\title{
In vivo dual cross-linking for identification of indirect DNA-associated proteins by chromatin immunoprecipitation
}

\author{
Ping-Yao Zeng 1,2, Christopher R. Vakoc ${ }^{3}$, Zhu-Chu Chen², Gerd A. Blobel3, \\ and Shelley L. Berger ${ }^{1}$ \\ 1The Wistar Institute, Philadelphia, PA, USA, ${ }^{2}$ The Cancer Research Institute, \\ Central South University, Changsha, Hunan, P.R. China, and ${ }^{3}$ Children's Hospital of \\ Philadelphia, Philadelphia, PA, USA
}

BioTechniques 41:694-698 (December 2006)

doi 10.2144/000112297

The chromatin immunoprecipitation (ChIP) assay (1) is a powerful technique to analyze protein-DNA interactions in a native chromatin context (2-4). ChIP is used to study a broad range of physiological processes including transcriptional regulation, DNA replication, and DNA repair. The assay is useful for a wide range of cells and tissues, including cells derived from yeasts, protozoa, Drosophila, and mammals. The ChIP assay typically comprises four steps: $(i)$ cross-linking proteins to DNA; (ii) chromatin fragmentation; (iii) protein precipitation; and (iv) quantitation (5). The initial crosslinking is used to covalently attach proteins within chromatin, to insure that DNA-protein complexes remain associated through the following steps. The DNA is broken into sizes ranging from approximately 500 bp (achieved by sonication) down to mononucleosomes (achieved by MNase digestion).

B

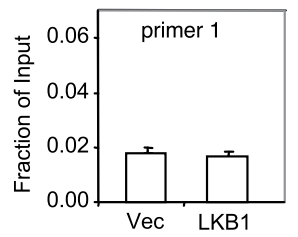

C

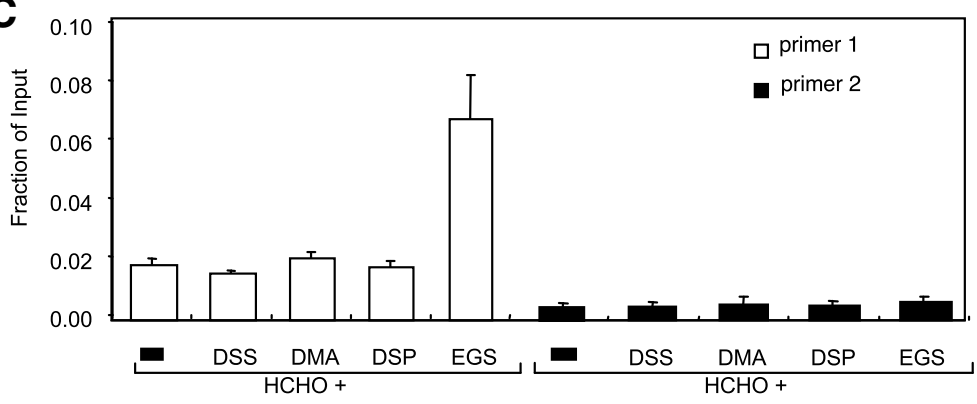

The DNA is then precipitated, commonly with an antibody to detect the protein of interest, but other means are also used, such as nickel-conjugated beads to precipitate a hexahistidine-tagged protein. The cross-links are then reversed to allow quantitation, using Southern blotting techniques, which directly measure the precipitated DNA, or by PCR, which is either semiquantitative (PCR followed by electrophoresis) or quantitative (PCR in real-time).

The most commonly used crosslinking reagent is formaldehyde (HCHO), because it is heat-reversible and is able to cross-link protein-DNA, protein-RNA, and protein-protein. Formaldehyde generates cross-links spanning approximately $2 \AA$ (although commercially available formaldehyde is polymerized, and, thus the actual cross-linking distance is unknown) (1) and is thus most useful to examine proteins directly bound to DNA, such as transcription factors, histones, and their covalent modifications (2-4). However, formaldehyde has a short cross-linking spacer arm and frequently is not effective to examine proteins that are indirectly associated with DNA, such as transcriptional coactivators and corepressors. Thus, it would be generally useful to identify a versatile cross-linking reagent that efficiently reveals proteins more distantly bound to DNA.

There have been recent reports of ChIP studies of indirectly bound cofactors using formaldehyde combined with a second cross-linking reagent bearing a longer spacer arm. The binding of the histone deacetylase Rpd3 in the yeast Saccharomyces cerevisiae was mapped using dimethyl adipimidate (DMA) (Figure 1) and formaldehyde $(6,7)$. A second approach using dimethyl 3,3'-dithiobispropionimidate (DTBP) plus formaldehyde
D

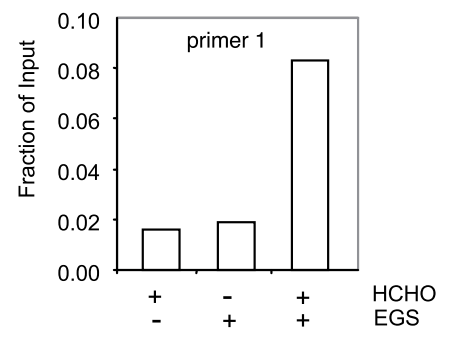

Figure 1. Chromatin immunoprecipitation (ChIP) assays testing different cross-linking agents to examine LKB1 at p21/WAF1. Immunoprecipitations were done with anti-LKB1 (gift from T. Makela, University of Helsinki; panels B and C) or anti-FLAG (Sigma; panel D). (A) The p21/WAF1 promoter shows the location of PCR primers relative to the p53 binding sites and the TATA box. (B) ChIP assay of FLAG-LKB1 at p21/WAF1. ChIP was done in cells stably expressing vector (Vec) alone or FLAG-LKB1 after formaldehyde cross-linking. (C) ChIP assay of FLAG-LKB1 using formaldehyde or combined formaldehyde/second agent as indicated. Primers 1 and 2 for PCR are indicated. (D) ChIP assay comparing formaldehyde alone, ethylene glycolbis[succinimidyl succinate] (EGS) alone, or combined formaldehyde/EGS. HCHO, formaldehyde; DSS, disuccinimidyl suberate; DMA, dimethyl adipimidate; DSP, dithiobis[succinimidyl propionate]. 


\section{Benchmarks}

revealed MTA3 present in the mammalian Mi-2/NuRD complex (8). Another group reported the using of disuccinimidyl glutarate (DSG) and formaldehyde to study the NFKB complex (9). Here, we report our investigation of combinations of cross-linkers to study cofactors that are refractory to ChIP analysis using formaldehyde alone. All of the protein-protein cross-linking reagents used in this study were purchased from Pierce Biotechnology (Rockford, IL, USA), and the formaldehyde was purchased from Sigma (St. Louis, MO, USA).

In one set of experiments we examined the role of a protein kinase, LKB1, in regulating p53-dependent genes. It was previously known that tumor suppressor LKB1 genetically interacts with p53 (10). We found that LKB1 physically associates with p53 in the nucleus (11), which led us to test whether LKB1 may function directly at promoters of genes regulated by $\mathrm{p} 53$. We transfected a plasmid encoding FLAG $^{\circledR}$ epitope-tagged LKB1 (pFLAG-LKB1), or a vector control, into Lkb1 null mouse embryo fibroblasts (MEFs) to establish stable cell lines (11). Using the conventional formaldehyde cross-linking based ChIP assay, we analyzed the LKB1 binding in the region of p53 binding sites upstream of the p21/WAF1 promoter (Figure 1A). We were unable to detect LKB1 binding to $\mathrm{p} 21 / \mathrm{WAF} 1$ compared with the vector control (Figure 1B). We then tested several cross-linking agents in combination with formaldehyde. These included DMA, disuccinimidyl suberate (DSS), dithiobis[succinimidyl propionate] (DSP), and ethylene glycolbis[succinimidyl succinate] (EGS) (Table 1). Each of these agents is membrane-permeable and has a longer spacer arm than formaldehyde (Table 1). The long-arm cross-linkers (DMA, DSS, DSP, or EGS) were separately incubated with the cell samples in phosphate-buffered saline (PBS) (Table 1) for 20-30 min at room temperature. Formaldehyde was then added at $1 \%$ of final concentration and incubated for a further $10 \mathrm{~min}$. The cross-linking reaction was quenched with $50 \mathrm{mM}$
glycine-PBS for $10 \mathrm{~min}$, and crosslinking was reversed by incubation at $65^{\circ} \mathrm{C}$ for $4-10 \mathrm{~h}$.

The PCR results show that as before (Figure 1B), formaldehyde alone did not yield a signal for LKB1 at the p21/ WAF1 promoter region, and neither DSS, DMA, nor DSP followed by formaldehyde indicated the presence of LKB1 (Figure 1C, left). However, dual cross-linking with EGS/formaldehyde revealed LKB1 at p21/WAF1 (Figure $1 \mathrm{C}$, left). The increase was between 3- and 4-fold compared with formaldehyde alone. As a control, LKB1 was not detected by EGS/formaldehyde dual cross-linking at the TATA box $5^{\prime}$ untranslated region (UTR) (Figure 1A) of the p21/WAF1 gene (Figure 1C, right). As a further control, we compared the ChIP efficiency using EGS alone and found that this treatment did not yield an LKB1-dependent signal (Figure 1D).

To verify the efficiency of this method in an unrelated system, we examined cofactors for the transcription factor GATA-1 at the locus control region (LCR) upstream of the murine $\beta$-globin locus in erythroid cells. GATA-1, which binds to DNA directly, can be detected easily by formaldehyde ChIP (Figure 2). However, signals for its non-DNA binding cofactor FOG-1 and the FOG-1-associated protein, MTA-2, were weak despite strong evidence by us and others that these proteins are important coregulators of GATA-1 in vivo (12). We found that formaldehyde alone yielded a very low ChIP signal for FOG-1 and nearly no signal for MTA-2 (Figure 2). In contrast, combined EGS/formaldehyde cross-linking increased the signals for these proteins 5-fold (FOG-1) and 8fold (MTA-2) (Figure 2). However, as expected, the combined cross-linking did not significantly increase the signal for GATA-1 (Figure 2). As a further control, we found that there was no ChIP signal for any of these proteins at the MyoD promoter (Figure 2), which is not regulated by these factors.

These data show that sequential cross-linking with EGS and formaldehyde is an effective method for ChIP analysis of multiple transcriptional cofactors that are refractory to analysis by conventional single formaldehyde cross-linking. We are currently uncertain why EGS appears to be a more robust protein-protein crosslinker in our studies of LKB1 than either DMA, DSS, or DSP. We note that EGS has the longest spacer arm of the reagents we tested. It is also possible that proteins vary in their reactivity with distinct types of cross-linkers. However, EGS appears to improve the signal-to-noise ratio in ChIPs in a broad variety of proteins that do not bind DNA directly (data not shown). In summary, EGS/formaldehyde dual cross-linking may prove to be generally useful for ChIP analysis of proteins that bind indirectly to DNA.

\section{ACKNOWLEDGMENTS}

We thank R. DePinho (Harvard University, Cambridge, MA) for the Lkb1 null MEFs. This work was supported by grants from the National Institutes of Health (NIH) CA07883106 (to S.L.B.) and DK54937 (to G.A.B). 


\section{Benchmarks}

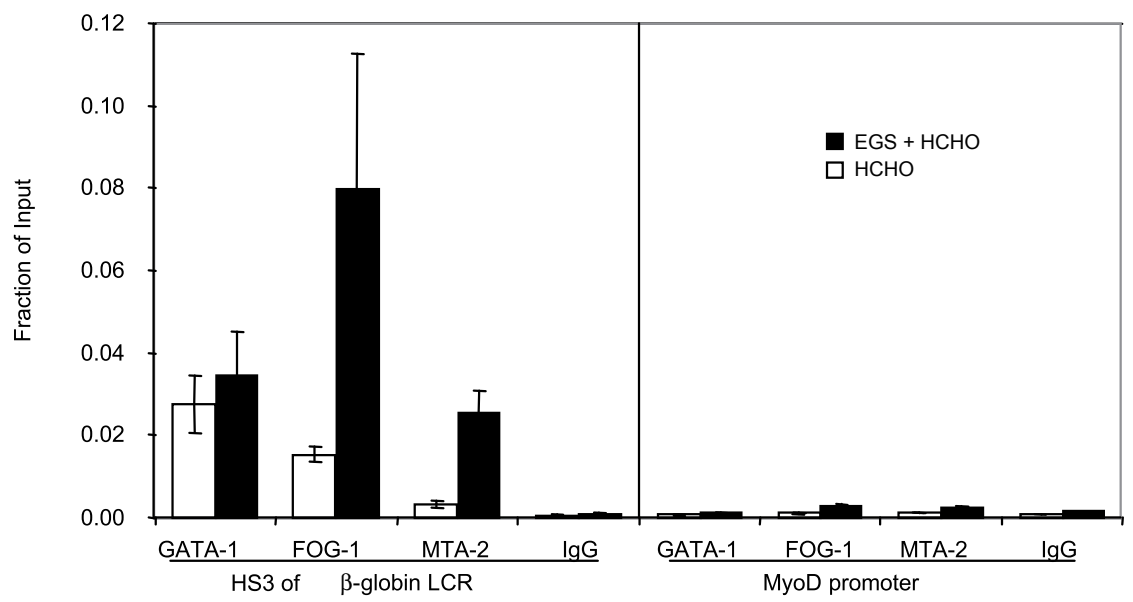

Figure 2. Chromatin immunoprecipitation (ChIP) assays at $\beta$-globin locus control region (LCR) and MyoD. Immunoprecipitations were done with anti-GATA-1 (sc265), anti-FOG-1 (sc9361), antiMTA-2 (sc9447) antibodies (Santa Cruz Biotechnology, Santa Cruz, CA, USA) or immunoglobulin $\mathrm{G}(\mathrm{IgG})$ as a negative control. Formaldehyde (HCHO) was compared with combined ethylene glycolbis[succinimidyl succinate] (EGS)/formaldehyde.

\section{COMPETING INTERESTS STATEMENT}

The authors declare no competing interests.

\section{REFERENCES}

1. Orlando, V., H. Strutt, and R. Paro. 1997. Analysis of chromatin structure by in vivo formaldehyde cross-linking. Methods 11:205214.

2. Evans, E., N. Sugawara, J.E. Haber, and E. Alani. 2000. The Saccharomyces cerevisiae Msh2 mismatch repair protein localizes to recombination intermediates in vivo. Mol. Cell 5:789-799.

3. Kuo, M.H. and C.D. Allis. 1999. In vivo cross-linking and immunoprecipitation for studying dynamic protein:DNA associations in a chromatin environment. Methods 19:425433.

4. Lo, A.W., J.M. Craig, R. Saffery, P. Kalitsis, D.V. Irvine, E. Earle, D.J. Magliano, and K.H. Choo. 2001. A 330 kb CENP-A binding domain and altered replication timing at a human neocentromere. EMBO J. 20:20872096.

5. Das, P.M., K. Ramachandran, J. vanWert, and R. Singal. 2004. Chromatin immunoprecipitation assay. BioTechniques 37:961-969.

6. Kurdistani, S.K. and M. Grunstein. 2003. In vivo protein-protein and protein-DNA crosslinking for genomewide binding microarray. Methods 31:90-95.

7. Kurdistani, S.K., D. Robyr, S. Tavazoie, and M. Grunstein. 2002. Genome-wide binding map of the histone deacetylase Rpd3 in yeast. Nat. Genet. 31:248-254.

8. Fujita, N., D.L. Jaye, M. Kajita, C. Geigerman, C.S. Moreno, and P.A. Wade. 2003. MTA3, a Mi-2/NuRD complex sub- unit, regulates an invasive growth pathway in breast cancer. Cell 113:207-219.

9. Nowak, D.E., B. Tian, and A.R. Brasier. 2005. Two-step cross-linking method for identification of NF-kappaB gene network by chromatin immunoprecipitation. BioTechniques 39:715-725.

10. Karuman, P., O. Gozani, R.D. Odze, X.C. Zhou, H. Zhu, R. Shaw, T.P. Brien, C.D. Bozzuto, et al. 2001. The Peutz-Jegher gene product LKB1 is a mediator of p53-dependent cell death. Mol. Cell 7:1307-1319.

11. Zeng, P.-Y. and S.L. Berger. LKB1 is recruited to the p21/WAF1 promoter by $\mathrm{p} 53$ to mediate transcriptional activation. Cancer Res. (In press).

12. Hong, W., M. Nakazawa, Y.Y. Chen, R. Kori, C.R. Vakoc, C. Rakowski, and G.A. Blobel. 2005. FOG-1 recruits the NuRD repressor complex to mediate transcriptional repression by GATA-1. EMBO J. 24:23672378.

Received 30 January 2006; accepted 7 September 2006.

Address correspondence to Shelley L. Berger, Gene Expression and Regulation Program, The Wistar Institute, 3601 Spruce Street, Philadelphia, PA 19104, USA. e-mail:berger@wistar.org

To purchase reprints of this article, contact: Reprints@BioTechniques.com 